\title{
RINGS THAT ARE FGC RELATIVE TO FILTERS OF IDEALS
}

\author{
by EROL BARBUT and WILLY BRANDAL
}

(Received 17th February 1987, revised 18th November 1987)

\section{Introduction}

All our rings will be commutative with identity not equal to zero. Also $R$ will always denote a ring. $\mathscr{F}$ is a filter of ideals of $R$ if $\mathscr{F}$ is a nonempty set of ideals of $R$ satisfying: if $I \in \mathscr{F}$ and $J$ is an ideal of $R$ with $I \subset J$, then $J \in \mathscr{F}$, and if $I, J \in \mathscr{F}$ then $I \cap J \in \mathscr{F}$. A Gabriel topology of $R$ is a filter of ideals $\mathscr{F}$ of $R$ satisfying: if $J \in \mathscr{F}$ and $I$ is an ideal of $R$ with $(I: x) \in \mathscr{F}$ for all $x \in J$, then $I \in \mathscr{F}$. See the B. Stenström text [6]. We say that a ring $R$ is an $F G C$ ring if every finitely generated $R$-module is a direct sum of cyclic $R$-modules. Use mspec $R$ for the set of all maximal ideals of $R$.

We shall use the following type of definition. Let $\mathscr{F}$ be a filter of ideals and let $\mathscr{P}$ be a ring property. Then $R$ is an $\mathscr{F}-\mathscr{P}$ ring if $R / I$ is a $\mathscr{P}$ ring for all $I \in \mathscr{F}-\{R\}$. The ring properties $\mathscr{P}$ that we shall use are FGC ring, Bezout ring, maximal ring and almost maximal ring. These are described in [2].

FGC rings have been characterized as a finite product of rings consisting of almost maximal Bezout domains, maximal valuation rings, and torch rings [2, Theorem 9.1]. Although we do not deal with torch rings, the principal goal of this paper is to characterize $\mathscr{F}$-FGC rings for certain filters of ideals, $\mathscr{F}$. We start with the classical case where $R$ is a domain and the filter of ideals is the set of all nonzero ideals, Theorem 1. We extend this to rings and certain filters of ideals in Theorem 2. Several examples are also included.

\section{Preliminaries}

Let $\mathscr{F}$ be a filter of ideals of $R$. Note that $R$ is an $\mathscr{F}$-FGC ring if and only if every finitely generated $\mathscr{F}$-pretorsion $R$-module decomposes into a direct sum of cyclic $R$ submodules. If $S$ is a multiplicatively closed subset of $R$ and $\mathscr{F}$ is a filter of ideals of $R$, then $\mathscr{F}_{S}$ denotes $\left\{I_{S}: I \in \mathscr{F}\right\}$. Then $\mathscr{F}_{S}$ is a filter of ideals of $R_{S}$. If $P \in \operatorname{spec} R$ and $S=R-P$, then we follow the common practice of denoting $R_{S}$ by $R_{p}$. Similarly use $\mathscr{F}_{\mathrm{P}}$ for $\mathscr{F}_{S}$ when $P \in \operatorname{spec} R$ and $S=R-P$. Beware that this is not the notation used in the B. Stenström text [6, p. 151].

Definition. Let $\mathscr{F}$ be a filter of ideals of $R$ and $\mathscr{P}$ a ring property. Then $R$ is a locally $\mathscr{F}-\mathscr{P}$ ring if $R_{M}$ is as $\mathscr{F}_{M^{-}} \mathscr{P}$ ring for all $M \in \operatorname{mspec} R$. 
In particular, we will use this definition for a locally $\mathscr{F}$-almost maximal ring, and a locally $\mathscr{F}$-valuation ring. If $I$ is an ideal of $R$, use $\operatorname{mspec}(I)$ for $\{M \in \operatorname{mspec} R: I \subset M\}$.

Definition. Let $\mathscr{F}$ be a filter of ideals of $R$. Then $R$ is an $\mathscr{F}$-local ring if

(1) $|\operatorname{mspec}(I)|<\infty$ for all $I \in \mathscr{F}$, and

(2) $|\operatorname{mspec}(P)|=1$ for all $P \in \mathscr{F} \hat{\cap} \operatorname{spec} R$.

Our definition of $\mathscr{F}$-local is inconsistent with the previously defined $\mathscr{F}-\mathscr{P}$ property. We allow this inconsistency because of our earlier use of $\mathscr{F}$-local as a generalization of $h$-local in [3], [4] and [5]. If $T$ is an $R$-module and $M \in \operatorname{mspec} R$, then use the notation

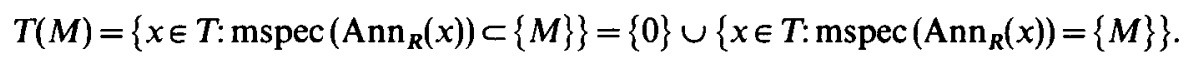

By [5, Theorem 1], if $\mathscr{F}$ is a filter of ideals of $R$, then $R$ is an $\mathscr{F}$-local ring if and only if $T=\bigoplus_{M \in \operatorname{mspec} R} T(M)$ for all $\mathscr{F}$-pretorsion modules $T$ if and only if $T=\bigoplus_{M \in \operatorname{mspec} R} T(M)$ for all cyclic $\mathscr{F}$-pretorsion modules $T$. There are a number of results relating to $\mathscr{F}$-local that have been proved for Gabriel topologies, which are in fact true for filters of ideals. Those that we shall be needing are [4, Lemma 1.1], [3, Theorem 1], and [2, Corollary $2.7(3)]$.

Proposition 1. Let $\mathscr{F}$ be a filter of ideals of $R$.

(1) Suppose $|\mathscr{F} \cap \operatorname{mspec} R| \leqq 1$. Then $R$ is an $\mathscr{F}-F G C$ ring if and only if $R$ is an $\mathscr{F}$ valuation ring and $R$ is an $\mathscr{F}$-almost maximal ring.

(2) If $R$ is an $\mathscr{F}$-FGC ring, then $R$ is an $\mathscr{F}$-Bezout ring.

Proof. [2, Theorem 4.5] and [2, Proposition 4.6].

The following is comparable to [2, Theorem 2.9], which is stated for integral domains with the classical torsion theory.

Proposition 2. Let $\mathscr{F}$ be a filter of ideals of $R$. Then $R$ is an $\mathscr{F}$-maximal ring if and only if $R$ is an $\mathscr{F}$-local ring and $R$ is a locally $\mathscr{F}$-maximal ring.

Proof. Suppose $R$ is an $\mathscr{F}$-maximal ring. We verify that $R$ is an $\mathscr{F}$-local ring. Let $I \in \mathscr{F}, I \neq R$. Since $R$ is an $\mathscr{F}$-maximal ring, $R / I$ is a linearly compact $R / I$-module. Using [2, Theorem 1.6], $|\mathrm{mspec}(I)|=|\mathrm{mspec} R / I|<\infty$. Suppose $P \in \mathscr{F} \cap \operatorname{spec} R$. Then $R / P$ is a linearly compact $R / P$-module. By [2, Corollary 1.7$],|\operatorname{mspec}(P)|=|\operatorname{mspec} R / P|=1$.

Now we verify that $R$ is a locally $\mathscr{F}$-maximal ring. Let $M \in \operatorname{mspec} R$ and $J \in \mathscr{F}_{M}$. There exists $I \in \mathscr{F}$ with $J=I_{M}$. By [5, Theorem 1] and [3, Theorem 1], $R / I \cong(R / I)_{M} \oplus$ $\left(\oplus_{M^{\prime} \in \operatorname{mspec} R-(M)}(R / I)_{M^{\prime}}\right)$. Also $(R / I)_{M} \cong R_{M} / J$, and $R_{M} / J$ is a linearly compact $R$-module by [2, Proposition 1.2 (2)], hence a linearly compact $R_{M}$-module.

Conversely, suppose $R$ is an $\mathscr{F}$-local ring and $R$ is a locally $\mathscr{F}$-maximal ring. Let 
$I \in \mathscr{F}$. Since $R$ is an $\mathscr{F}$-local ring, $|\operatorname{mspec}(I)|<\infty$, and by [5, Theorem 1] and [3, Theorem 1], $R / I \cong \oplus_{M \in \operatorname{mspec} R} R_{M} / I_{M}$. If $M \in \operatorname{mspec} R-\operatorname{mspec}(I)$, then $R_{M} / I_{M} \cong\{0\}$. Since $R$ is a locally $\mathscr{F}$-maximal ring, $R_{M} / I_{M}$ is a linearly compact $R_{M}$-module. By [2, Corollary 2.7 (3)], $R_{M} / I_{M}$ is a linearly compact $R$-module. Combining, we see that $R / I$ is a finite direct sum of linearly compact $R$-modules. By [2, Proposition $1.2(4)], R / I$ is a linearly compact $R$-module. Hence $R$ is an $\mathscr{F}$-maximal ring.

Let $S$ be a multiplicatively closed subset of $R$ and let $\mathscr{F}$ be a filter of ideals of $R$. If $R$ is an $\mathscr{F}$-FGC ring then $R_{\mathrm{S}}$ is an $\mathscr{F}_{\mathrm{S}}$-FGC ring and if $R$ is an $\mathscr{F}$-Bezout ring then $R_{S}$ is an $\mathscr{F}_{s}$-Bezout ring. Also if $R$ is an $\mathscr{F}$-Bezout ring, then $R$ is a locally $\mathscr{F}$-valuation ring.

\section{The domain case}

Let $R$ be a domain. Use the notation $\mathscr{N}_{R}$ for the set of all nonzero ideals of $R$. Then $\mathcal{N}_{R}$ is a Gabriel topology of $R$, and the classical torsion theory of $R$ is $\mathscr{N}_{R}$-torsion. Note that if $R$ is not a domain, we shall not use the notion $\mathscr{N}_{R}$ because in that case the set of all nonzero ideals of $R$ is not necessarily a filter of ideals of $R$.

Suppose that $R$ is a domain and $S$ a multiplicatively closed subset of $R$. Note that if $R$ is an $\mathscr{N}_{R}$-FGC ring, then $R_{S}$ is an $\mathscr{N}_{R_{s}}$-FGC ring.

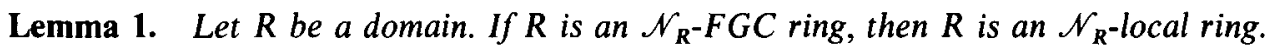

Proof. We have $|\operatorname{mspec}(P)|=1$ for all $P \in \mathcal{N}_{R} \cap \operatorname{spec} R$. This follows by an argument similar to that of S. Wiegand [2, proof of Theorem 5.6].

Suppose $I \in \mathcal{N}_{R}$. We want to show that $|\operatorname{mspec}(I)|<\infty$. Suppose $I \neq R$. Then $R / I$ is an FGC ring. By [2, Theorem 8.5], minspec $R / I$ is finite (where minspec $R$ denotes the set of all minimal prime ideals of $R$ ). By the preceding paragraph, $|\operatorname{mspec}(I)| \leqq$ $\mid$ minspec $R / I \mid<\infty$. By definition, $R$ is an $\mathscr{N}_{R^{-}}$-local ring.

Lemma 2. Let $R$ be a domain. Then $R$ is an $\mathcal{N}_{R^{-}}$almost maximal ring if and only if $R$ is an $\mathscr{N}_{R^{-}}$maximal ring.

Proof. If $R$ is an $\mathscr{N}_{R}$-maximal ring, then $R$ is an $\mathscr{N}_{R}$-almost maximal ring by [2, Proposition 1.2 (2)]. Conversely, suppose $R$ is an $\mathscr{N}_{R}$-almost maximal ring. If $R$ is a field, then we are done. If $R$ is not a field, then for all $I \in \mathscr{N}_{R}$ there exists $J \in \mathscr{N}_{R}$ with $J \varsubsetneqq I$, and it follows that $R$ is an $\mathcal{N}_{R}$-maximal ring.

Theorem 1. Let $R$ be a domain. The following statements are equivalent.

(1) $R$ is an $\mathcal{N}_{R^{-}}$FGC ring.

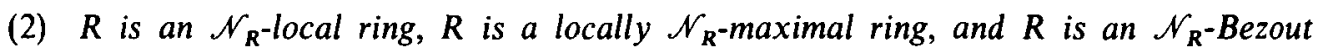
ring.

(3) $R$ is an $\mathscr{N}_{R^{-}}$local ring, $R$ is a locally $\mathscr{N}_{R^{-}}$almost maximal ring, and $R$ is an $\mathcal{N}_{R}$-Bezout ring. 
(4) $R$ is an $\mathscr{N}_{R}$-maximal ring and $R$ is an $\mathscr{N}_{R}$-Bezout ring.

(5) $R$ is an $\mathcal{N}_{R^{-}}$-almost maximal ring and $R$ is an $\mathcal{N}_{R^{-}}$-Bezout ring.

Proof. (1) $\Rightarrow$ (3). Suppose $R$ is an $\mathscr{N}_{R}$-FGC ring. By Proposition $1, R$ is an $\mathcal{N}_{R}$-Bezout ring. By Lemma $1, R$ is an $\mathcal{N}_{R^{-}}$local ring. Let $M \in \operatorname{mspec} R$. Then $R_{M}$ is an $\mathscr{N}_{R_{M}}$-FGC ring. By proposition $1, R_{M}$ is an $\mathscr{N}_{R_{M}}$-almost maximal ring. This means that $R$ is a locally $\mathscr{N}_{R^{-a l m o s t}}$ maximal ring.

(3) $\Rightarrow(2)$. Lemma 2 .

(2) $\Rightarrow(1)$. Suppose $R$ is an $\mathscr{N}_{R}$-local ring, $R$ is a locally $\mathscr{N}_{R^{-}}$-maximal ring, and $R$ is an $\mathscr{N}_{R}$-Bezout ring. Let $A$ be a finitely generated $\mathscr{N}_{R}$-torsion $R$-module. Since $R$ is an $\mathscr{N}_{R}$-local ring, $A=\oplus_{M \in \operatorname{mspec} R} A(M)$. Since $A$ is a finitely generated $R$-module, there exist $M_{1}, \ldots, M_{m} \in \operatorname{mspec} R$ with $A=\bigoplus_{i=1}^{m} A\left(M_{i}\right)$. Let $S=R_{M_{i}}$, and use [4, Lemma 1.1], to show that $A\left(M_{i}\right)$ is a finitely generated $\mathscr{N}_{S}$-torsion $S$-module. Let $M \in \operatorname{mspec} R$. Then $R_{M}$ is an $\mathscr{N}_{R_{M}}$-valuation ring. Also $R_{M}$ is an $\mathscr{N}_{R_{M}}$-maximal ring. By [2, Theorem 4.2], $R_{M}$ is an FGC ring. Then each $A\left(M_{i}\right)$ decomposes into a direct sum of cyclic $R_{M_{i}}$-submodules. Use [2, Corollary $2.7(3)]$, to show that $A\left(M_{i}\right)$ decomposes into a direct sum of cyclic $R$-submodules. It follows that $R$ is an $\mathscr{N}_{R}$-FGC ring.

$(2) \Leftrightarrow(4)$. Proposition 2 .

$(4) \Leftrightarrow(5)$. Lemma 2 .

The reader might suspect that if $\mathscr{F}$ is a filter of ideals of $R$, then $R$ is an $\mathscr{F}$-maximal ring if and only if $R$ is an $\mathscr{F}$-almost maximal ring. This is not the case, for let $R$ be the ring of integers and let $\mathscr{F}_{0}$ be the set of all ideals of $R$.

As a special case of the above theorem, we have that if $R$ is an $\mathscr{N}_{R}$-FGC domain, then $R$ is an $\mathscr{N}_{R}$-maximal ring. However, $R$ is an $\mathscr{F}$-FGC ring in general does not imply that $R$ is an $\mathscr{F}$-maximal ring. For a counterexample take the example of the preceding paragraph. We just had two statements which have trivial counterexamples, involving a filter of ideals $\mathscr{F}$ with the property that there is a minimal element in $\mathscr{F}$ which is a prime ideal. We have encountered this situation several times in the past, and we are uncertain as to why such primes cause difficulties.

Of course an FGC domain is an $\mathcal{N}_{R}$-FGC ring. Besides the well known FGC domains, there is another class of $\mathscr{N}_{R}$-FGC domains. Namely, if $R$ is a Dedekind domain that is not a PID, then $R$ is an $\mathscr{N}_{R}$-FGC ring and $R$ is not an FGC ring. Note also that such a Dedekind domain is not a Bezout domain, yet it is an $\mathscr{N}_{R}$-Bezout ring.

A domain $R$ is said to be a Prüfer domain if $R_{M}$ is a valuation domain for all $M \in \operatorname{mspec} R$. It is well known that a Bezout domain is a Prüfer domain. E. Matlis [2, Theorem 5.1] has proved that if $R$ is an $\mathscr{N}_{R}$-local locally $\mathscr{N}_{R}$-maximal Prüfer domain, then $R$ is $\mathscr{N}_{R}$-FGC ring. This is closely related to Theorem 1 part $(2) \Rightarrow(1)$. A Prüfer domain need not be an $\mathscr{N}_{R}$-Bezout ring. It is not difficult to show that an $\mathscr{N}_{R}$-local Prüfer domain is an $\mathscr{N}_{R}$-Bezout ring (use [2, Proposition 3.8]). Thus in Theorem 1 statements (2), (3), (4) and (5) we could replace " $\mathcal{N}_{R}$-Bezout" by "Prüfer". 


\section{Generalizing the domain case}

Let $P \in \operatorname{spec} R$. Use the notation $\mathscr{F}_{(P)}$ for the filter, $\{I: I$ is an ideal of $R$ and $I \supsetneqq P\}$, of ideals of $R$. This should not be confused with a similar notation of the B. Stenström text $\left[6\right.$, p. 151] which denotes $\{I: I$ is an ideal of $R$ and $I \subset P\}$. The filter, $\mathscr{F}_{(P)}$ need not be a Gabriel topology as the following example shows.

Example. Let $k$ be a field with $X$ and $Y$ indeterminates over $k$. Let $R$ be the polynomial ring $k[X, Y]$. Let $P=R X \in \operatorname{spec} R$ and $J=R X+R Y$. Then $J \in \mathscr{F}_{(P)}$, and $J^{2} \notin \mathscr{F}_{(P)}$. Hence $\mathscr{F}_{(P)}$ is not a Gabriel topology of $R$.

Note that Theorem 1 still remains true if we replace $\mathscr{N}_{R}$ by $\mathscr{F}_{(P)}$ for $P \in \operatorname{spec} R$, in a ring $R$. Before presenting the main generalization of Theorem 1 , we need a few preliminaries.

Proposition 3. The following statements are equivalent.

(1) $R$ is an almost maximal Bezout ring.

(2) Either $R$ is an almost maximal Bezout domain or $R$ is a finite product of rings each of which is a maximal valuation ring.

Proof. $\quad(1) \Rightarrow(2)$. Suppose $R$ is an almost maximal Bezout ring and $R$ is not a domain. Then there exist nonzero $x, y \in R$ with $x y=0$. Since $R / R x$ and $R / R y$ are maximal rings, they are a finite product of local rings by [2, Theorem 1.6]. Recall that a local Bezout ring is a valuation ring [2, Lemma 3.6]. It follows that $R$ has only finitely many maximal ideals, $R$ has the same finite number of minimal prime ideals, and each minimal prime ideal of $R$ is a subset of only one maximal ideal of $R$. Suppose $\left\{P_{1}, \ldots, P_{n}\right\}$ is the set of minimal prime ideals of $R$. The minimal prime ideals are pairwise comaximal and $R / \bigcap \operatorname{spec} R \cong \bigoplus_{j=1}^{n} R / P_{j}$. Idempotents modulo the prime radical can be lifted to $R$. Thus $R=\bigoplus_{j=1}^{n} R_{j}$, where each $R_{j}$ is a ring with a unique maximal ideal. Hence each $R_{j}$ must be a maximal valuation ring.

$(2) \Rightarrow(1)$. Straightforward.

Corollary. If $R$ is an almost maximal Bezout ring, then $R$ is an FGC ring.

Proof. Proposition 3 and [2, Theorem 9.1].

Definitions. Let $\mathscr{F}$ be a filter of ideals of $R$. Then $R$ is an intersection $\mathscr{F}$-almost maximal ring if for all $I \in \mathscr{F}$ there exists a finite set $\left\{I_{1}, \ldots, I_{n}\right\}$ of pairwise comaximal ideals of $R$ with $I=\bigcap_{j=1}^{n} I_{j}$ and every proper $R$-homomorphic image of $R / I_{j}$ is a linearly compact $R$-module for all $j=1, \ldots, n$. Also $R$ is an intersection $\mathscr{F}$-maximal ring if for all $I \in \mathscr{F}$ there exists a finite set $\left\{I_{1}, \ldots, I_{n}\right\}$ of pairwise comaximal ideals of $R$ with $I=\bigcap_{j=1}^{n} I_{j}$ and $R / I_{j}$ is a linearly compact $R$-module for all $j=1, \ldots, n$. 
Definition. Let $\left\{P_{1}, \ldots, P_{n}\right\}$ be a finite set of pairwise comaximal prime ideals of $R$. Then the product of $\mathscr{F}_{\left(P_{1}\right)}, \ldots, \mathscr{F}_{\left(P_{n}\right)}$ is $\left\{I_{1} \ldots I_{n}: I_{j} \in \mathscr{F}_{\left(P_{j}\right)}\right.$ for all $\left.j=1, \ldots, n\right\}$.

Let $\mathscr{F}$ be the product of $\mathscr{F}_{\left(P_{1}\right)}, \ldots, \mathscr{F}_{\left(P_{n}\right)}$ If $I_{1}, \ldots, I_{n} \in \mathscr{F}$ with $I_{j} \in \mathscr{F}_{\left(P_{j}\right)}$, then $\left\{I_{1}, \ldots, I_{n}\right\}$ is a pairwise comaximal set of ideals of $R$, and $I_{1} \ldots I_{n}=\bigcap_{j=1}^{n} I_{j}$. In other words, $\mathscr{F}=\left\{\bigcap_{j=1}^{n} I_{j}: I_{j} \in \mathscr{F}_{\left(P_{j}\right)}\right.$ for all $\left.j=1, \ldots, n\right\}$. It follows that $\mathscr{F}$ is a filter of ideals of $R$. We also have that $\mathscr{F}$ is the smallest filter of ideals of $R$ that has $\bigcup_{j=1}^{n} \mathscr{F}_{\left(P_{j}\right)}$ as a subset.

Theorem 2. Let $\left\{P_{1}, \ldots, P_{n}\right\}$ be a finite set of pairwise comaximal prime ideals of $R$, and let $\mathscr{F}$ be the product of $\mathscr{F}_{\left(P_{1}\right)}, \ldots, \mathscr{F}_{\left(P_{n}\right)}$. The following statements are equivalent.

(1) $R$ is an $\mathscr{F}-F G C$ ring.

(2) $R$ is an $\mathscr{F}_{\left(P_{j}\right)}-F G C$ ring for all $j=1, \ldots, n$.

(3) $R$ is an intersection $\mathscr{F}$-almost maximal ring and $R$ is an $\mathscr{F}$-Bezout ring.

(4) $R$ is an intersection $\mathscr{F}$-maximal ring and $R$ is an $\mathscr{F}$-Bezout ring.

Proof. $(1) \Rightarrow(2)$. Suppose $R$ is an $\mathscr{F}-\mathrm{FGC}$ ring. Since $\mathscr{F} \supset \mathscr{F}_{\left(P_{j}\right)}, R$ is an $\mathscr{F}_{\left(P_{j}\right)}-\mathrm{FGC}$ ring for all $j=1, \ldots, n$.

(2) $\Rightarrow(3)$. Suppose $R$ is an $\mathscr{F}_{\left(P_{j}\right)}$-FGC ring for all $j=1, \ldots, n$. Let $I \in \mathscr{F}$ and assume $I \neq R$. Then $I=\bigcap_{j=1}^{n} I_{j}$ for some $I_{j} \in \mathscr{F}_{\left(P_{j}\right)}$. By Theorem $1, R$ is an $\mathscr{F}_{\left(P_{j}\right)}$-almost maximal ring and $R$ is an $\mathscr{F}_{\left(P_{j}\right)}$-Bezout ring. Thus every proper $R$-homomorphic image of $R / I_{j}$ is a linearly compact $R$-module. This verifies that $R$ is an intersection $\mathscr{F}$-almost maximal ring. Also $R / I_{j}$ is a Bezout ring for all $I_{j} \neq R$. So $R / I=R / \bigcap_{j=1}^{n} I_{j} \cong \bigoplus_{j=1}^{n} R / I_{j}$ is also a Bezout ring.

$(3) \Rightarrow(4)$. This follows from Lemma 2.

(4) $\Rightarrow(1)$. Suppose $R$ is an intersection $\mathscr{F}$-maximal ring and $R$ is an $\mathscr{F}$-Bezout ring. Let $A$ be a finitely generated $\mathscr{F}$-pretorsion $R$-module. If $\mathscr{F}=\{R\}$, then $A=\{0\}$ and $A$ decomposes into a direct sum of cyclic $R$-submodules. Suppose $\mathscr{F} \neq\{R\}$. Then there exists $I \in \mathscr{F}-\{R\}$ with $I A=\{0\}$. There exists a finite set $\left\{I_{1}, \ldots, I_{m}\right\}$ of pairwise comaximal proper ideals of $R$ with $I=\bigcap_{i=1}^{m} I_{j}$ and $R / I_{j}$ a linearly compact $R$-module for all $j=1, \ldots, m$. Also $R / I=R / \bigcap_{j=1}^{m} I_{j} \cong \bigoplus_{j=1}^{m} R / I_{j}$. But $I_{j} \supset I \in \mathscr{F}$ implies $I_{j} \in \mathscr{F}$. Hence each $R / I_{j}$ is a maximal Bezout ring and an $\mathrm{FGC}$ ring. Therefore $R / I$ is a finite direct sum of FGC rings, and $R / I$ is an FGC ring. View $A$ as a finitely generated $R / I$ module. Then $A$ decomposes into a direct sum of cyclic $R / I$-submodules. Hence $A$ decomposes into a direct sum of cyclic $R$-submodules. By definition $R$ is an $\mathscr{F}$-FGC ring.

We present three examples of $\mathscr{F}$-FGC rings related to the generalization of Theorem 1.

Example. Let $R_{1}, \ldots, R_{n}$ be indecomposable FGC rings. Then each $R_{\mathrm{i}}$ has a unique minimal prime ideal $I_{i}$ by $\left[2\right.$, Theorem 9.1]. Let $R=\bigoplus_{j=1}^{n} R_{j}$ and let 


$$
P_{i}=R_{1} \oplus \cdots \oplus R_{i-1} \oplus I_{i} \oplus R_{i+1} \oplus \cdots \oplus R_{n} .
$$

Then $\left\{P_{1}, \ldots, P_{n}\right\}$ is a finite set of pairwise comaximal prime ideals of $R$ and this is also the set of all minimal prime ideals of $R$. Let $\mathscr{F}$ be the product of $\mathscr{F}_{\left(P_{1}\right)}, \ldots, \mathscr{F}_{\left(P_{n}\right)}$. Each $R_{i}$ is an $\mathscr{F}_{\left(P_{i}\right)}$-FGC ring. By Theorem $2, R$ is an $\mathscr{F}$-FGC ring and $R$ is an intersection $\mathscr{F}$-maximal ring.

Example. All groups of this example are assumed abelian, and we follow the terminology and notation of [1]. Let $Z$ be the set of integers viewed as an additive group with the usual total ordering. Let $H_{1}, \ldots, H_{n}$ be totally ordered groups. For $i=1, \ldots, n$ define $G_{i}=H_{i} \oplus Z$ ordered lexicographically. Then each $G_{i}$ is a totally ordered group. Define $I_{i}=\left\{g \in G_{i}: g=(h, k), h \in H_{i}, k \in Z\right.$, and $\left.h>0\right\}$. Then $I_{i} \in \operatorname{spec} G_{i}$. Define $G=\bigoplus_{i=1}^{n} G_{i}$ with the product ordering. Then $G$ is a lattice ordered group. Define

$$
J_{i}=\left(G_{1}\right)_{+} \oplus \cdots \oplus\left(G_{i-1}\right)_{+} \oplus I_{i} \oplus\left(G_{i+1}\right)_{+} \oplus \cdots \oplus\left(G_{n}\right)_{+} .
$$

Then $J_{i} \in \operatorname{spec} G$. By the Krull-Kaplansky-Jaffard-Ohm construction [1, Theorem 1.9], there exists a Bezout domain $R$ with divisibility group lattice isomorphic to $G$. Let $P_{i} \in \operatorname{spec} R$ with $P_{i}$ corresponding to $J_{i}\left[1\right.$, Proposition 1.2]. Then $\left\{P_{1}, \ldots, P_{n}\right\}$ is a finite set of pairwise comaximal prime ideals of $R$, and $R / P_{i}$ is a discrete valuation domain. Let $\mathscr{F}$ be the product of $\mathscr{F}_{\left(P_{1}\right)}, \ldots, \mathscr{F}_{\left(P_{n}\right)}$. Then $R$ is an $\mathscr{F}_{\left(P_{i}\right)}-$ FGC ring for all $i=1, \ldots, n$. By Theorem $2, R$ is an $\mathscr{F}$-FGC ring and $R$ is an intersection $\mathscr{F}$-maximal ring.

Example. Let $N$ be the set of positive integers and let $F$ be a field. Define $R$ to be the ring $F^{N}$ and write elements of $R$ as infinite tuples $\left\langle x_{n}\right\rangle$ where $x_{n} \in F$ for all $n \in N$. Define $M_{k}=\left\{\left\langle x_{n}\right\rangle \in R: x_{k}=0\right\}$ for $k \in N$. Then $\left\{M_{k}\right\}_{k \in N} \subset \operatorname{mspec} R$. Define $\mathscr{F}=$ $\left\{\bigcap_{k \in X} M_{k}: X\right.$ is a finite subset of $\left.N\right\}$. Then $R$ is an $\mathscr{F}$-FGC ring and $R$ is an intersection $\mathscr{F}$-maximal ring. However, $\mathscr{F}$ is not a product of $\mathscr{F}_{\left(P_{1}\right)}, \ldots, \mathscr{F}_{\left(P_{n}\right)}$ for any $P_{i}$.

If $R$ is an $\mathscr{F}$-FGC ring, then it does not follow that $R$ is an intersection $\mathscr{F}$-almost maximal ring. For example, if $R$ is a torch ring [2] and $\mathscr{F}_{0}$ is the set of all ideals of $R$, then $R$ is an $\mathscr{F}_{0}$-FGC ring, but $R$ is not an intersection $\mathscr{F}_{0}$-almost maximal ring. This example illustrates our emphasis on the two types of indecomposable FGC rings: almost maximal Bezout domains and maximal valuation rings. We have not yet dealt with the third type of indecomposable FGC ring: torch rings. This could be the topic of future research.

\section{REFERENCES}

1. W. Brandal, Constructing Bezout domains, Rocky Mountain J. Math. 6 (1976), 383-399. MR 54 \# 2644.

2. W. Brandal, Commutative rings whose finitely generated modules decompose (Lecture Notes in Mathematics, Springer-Verlag, 723 Berlin, 1979). MR 80g \# 13003.

3. W. Brandal, Locally injective torsion modules, Rocky Mountain J. Math. 16 (1986), 17-22. 
4. W. Brandal and E. Barbut, Torsion theories over commutative rings, J. Algebra 101 (1986), 136-150.

5. W. Brandal and E. Barbut, Decomposing torsion modules, Communications in Algebra 15(6) (1987), 1109-1117.

6. B. Stenström, Rings of Quotients (Die Grundlehren der mathematischen Wissenschaften, 217 Springer-Verlag, Berlin, 1975). MR 52 \# 10782.

Department of Mathematics and Statistics

UNIVERSITY OF IDAHO

Moscow, ID 83843

USA 